\title{
Inferior Part of Rectus Abdominis Muscle Flap Outcomes after Abdominoperineal Resection: A Case Series Pilot Study
}

\author{
Mahdi Alemrajabi ${ }^{1}$, Morteza Khavanin Zadeh ${ }^{2}$, Nima Hemmati ${ }^{1}$, Behrouz Banivaheb ${ }^{1}$, \\ Fatemeh Alemrajabi ${ }^{1}$, Sepideh Jahanian ${ }^{1}$, Mohammad Bahadoram ${ }^{1}$, Maedeh Barahman ${ }^{{ }^{*}}$
}

\footnotetext{
1. Firoozgar Clinical Research Development Center (FCRDC), Firoozgar hospital, Iran University of Medical Sciences (IUMS), Tehran, Iran.

2. Hasheminejad Kidney Center (HKC), Iran University of Medical Sciences (IUMS), Tehran, Iran.
}

*Corresponding Author:

Maedeh Barahman

Firoozgar Clinical Research Development Center (FCRDC), Firoozgar hospital, Iran University of Medical Sciences (IUMS), Tehran, Iran.

Tel.: +989125444736

Email: brahman.m@iums.ac.ir

Received: 22 Jul 2021

Revised: 25 Aug 2021

Accepted: 19 Sep 2021

\section{ABSTRACT}

\section{BACKGROUND}

The standard surgical treatment for low rectal cancer is abdominoperineal resection (APR). Comparing to primary closure, immediate flap reconstruction has shown to have good outcomes. We aimed to assess the inferior rectus abdominis muscle flap complications after APR surgery, a new method of reconstruction.

\section{METHODS}

This study was conducted from 2014 to 2016 in a single center in Firoozgar Hospital, Tehran, Iran. Eighteen patients who underwent pelvic floor closure with inferior part of abdominis rectus musculofascial flap were included enrolled. The sampling method used in this study was census. All patients had distal rectoanal malignancies. A checklist including age, gender, tumor location, complications after surgery, tumor type, length of hospital stay, length of operation, neoadjuvant chemotherapy and neoadjuvant radiotherapy history was filled for all patients.

\section{RESULTS}

Among 18 participants, $27.8 \%$ were female. The mean age of participants was $58.28 \pm 17.86 \mathrm{yr}$ (minimum of 19 and the maximum of $89 \mathrm{yr}$ ). The pathology of the tumor in all but one of the cases was adenocarcinoma (94.4\%). The overall complication rate after surgery was $27.8 \%$. In total, $80 \%$ received neoadjuvant chemoradiotherapy. In 12 months follow-up 2 patients needed reoperation.

\section{CONCLUSION}

Inferior part of rectus abdominis muscle flap was a reliable and comparable means of reconstruction after APR surgery with low rate of complications and mortality.

\section{KEYWORDS}

Abdominoperineal resection; Rectaloanal malignancies; immediate flap reconstruction

Please cite this paper as:

Alemrajabi M., Khavanin Zadeh M., Hemmati N., Banivaheb B., Alemrajabi F., Jahanian S., Bahadoram M., Barahman M. Inferior Part of Rectus Abdominis Muscle Flap Outcomes after Abdominoperineal Resection: A Case Series Pilot Study. World J Plast Surg. 2021;10(2):104-110.

doi: 10.29252/wjps.10.3.104 


\section{INTRODUCTION}

The standard surgical treatment for low rectal cancer is abdominoperineal resection (APR). Usually, extensive resections produce an extensive pelvic defect that present complications including wound closure challenges, infections and dehiscence. Comparing to primary closure, it had been shown that immediate flap reconstruction has fewer complications ${ }^{1-6}$.

A large, non-collapsible dead space in pelvis is associated with wound-healing problems complications. Pelvis irridations also adds to the problem. Surgeons suggested different types of myocutaneous flap techniques to provide coverage for blank space in perineum ${ }^{1,2,7,8}$.

Myocutaneous flaps provide bulky and healthy tissues which are well vascularized to fill the empty space of perineum, thus improving better wound healing and decreased infection rates. It can also provide better access for immune system agents to the area and increase oxygenation ${ }^{8}$.

Different options for pelvic reconstruction include pedicled vertical rectus abdominis myocutaneous (VRAM) flaps, local V to Y advancement flaps and pedicled gracilis muscle flaps. There are several pros and cons for the existing flaps. The pedicled VRAM flaps has fewer rate of complications than primary wound closure. A decrease of perineal morbidity has been also seen in VRAM flaps and it also had a well long term survival. Compared to gracilis flaps, VRAM flaps have shown better results ${ }^{9-17}$. VRAM flap method use is limited by some factors including previous abdominal surgery, positioning, scarring and etc'.

$\mathrm{V}$ to $\mathrm{Y}$ advancement flaps make less abdominal wall morbidity comparing VRAM flaps and the flap size is less bulky in comparison ${ }^{18,19}$. Although gracilis flaps may not be applicable to patients with larger pelvic defects ${ }^{20}$.

There is still no consensus regarding the best option for pelvic space filling. Thus, we aimed to assess the inferior rectus abdominis muscle flap complications after APR surgery.

\section{METHODS AND MATERIALS}

Among 204 cases of proctectomy in 2014 to 2016, in Firoozgar Hospital, Iran University of Medical Sciences, Tehran, Iran; 38 underwent abdominoperineal resection and 18 pelvic floor closure with inferior part of rectus abdominis flap reconstruction. Inclusion criteria were as follow; all patients had distal recto-anal malignancies with sphincter involvement. Female patients should have had a prior history of hysterectomy. Prior to surgery, patients underwent colonoscopy and biopsy. A computed tomography (CT) scan was performed for all patients to assess lymph node involvement and distant metastasis.

Exclusion criteria were; 1) prior abdominal surgery or abdominal wall defects, 2) distant metastasis and terminal cancer, 3) adjacent organ involvement requiring a wide resection, 4) no consent to take part in the study. Finally, 18 patients underwent pelvic floor closure with inferior part of abdominis rectus muscle flap.

A checklist including age, gender, tumor location, complications after surgery, tumor type, length of hospital stay, length of operation, neoadjuvant chemotherapy and neoadjuvant radiotherapy history was filled for all patients. Patients were followed for complications in one-year period after surgery. The complications were defined as; 1) dehiscence, dermal separation more than one third of the wound but without infection, 2) abscess, a purulent collection requiring drainage, 3) flap loss, necrosis of at least one third of flapped tissue, 4) infection, a mucopurulent discharge from the wound, 5) prolonged healing, absence of wound closure at $3 \mathrm{wk}$ from surgery, 5) obstruction, nausea and vomiting with non-presenting feces in the colostomy with abdominal distension, 6) cellulitis, inflammation of the tissue around the wound requiring antibiotic treatment.

\section{Surgical technique}

The method of reconstruction was Alem's reconstruction method described in a previous case report ${ }^{21}$. In brief, thorough a midline incision, abdominal cavity was accessed. The inferior part of the right rectus muscle with its posterior fascia was dissected carefully down to its pubic origin (Figure 1). Appropreate location was selected to place colostomy on the left side (Figure 2). Maximum effort was made to preserve the perforating and inferior epigastric arteries (Figure 3). The flap was then placed in the pelvic vacant space and fixed with loose stiches (usually vicryl 3-0) without any tension (Figures 4 and 5). After placement of end colostomy, 


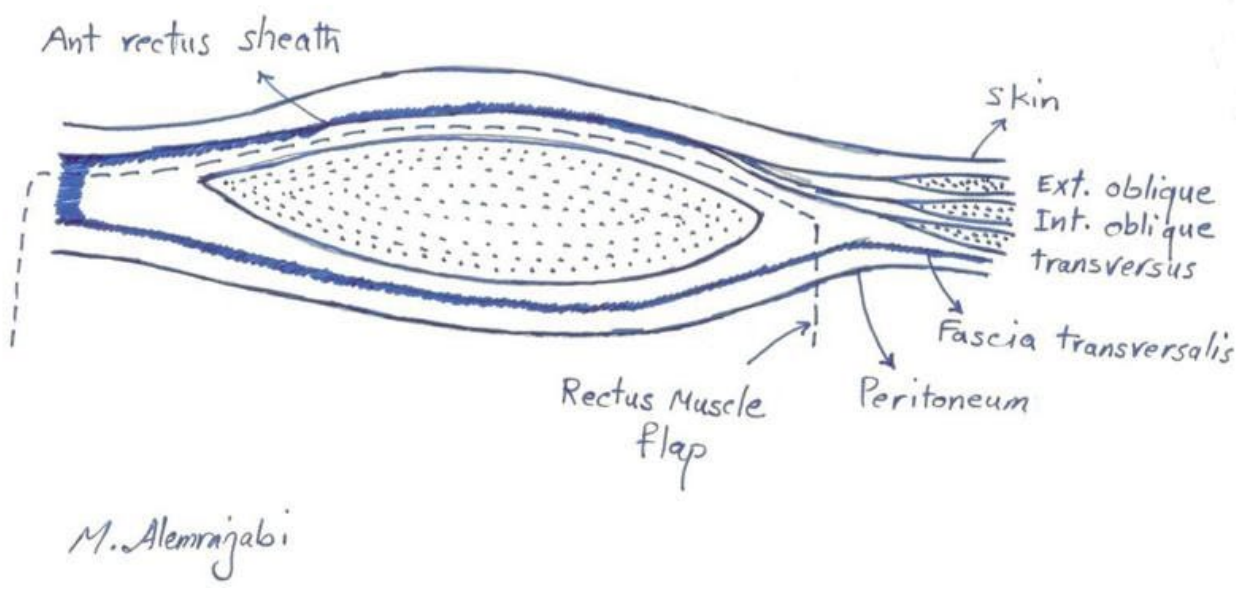

Fig. 1: The skin and anterior fascia was dissected from the bulk of rectus abdominis muscle

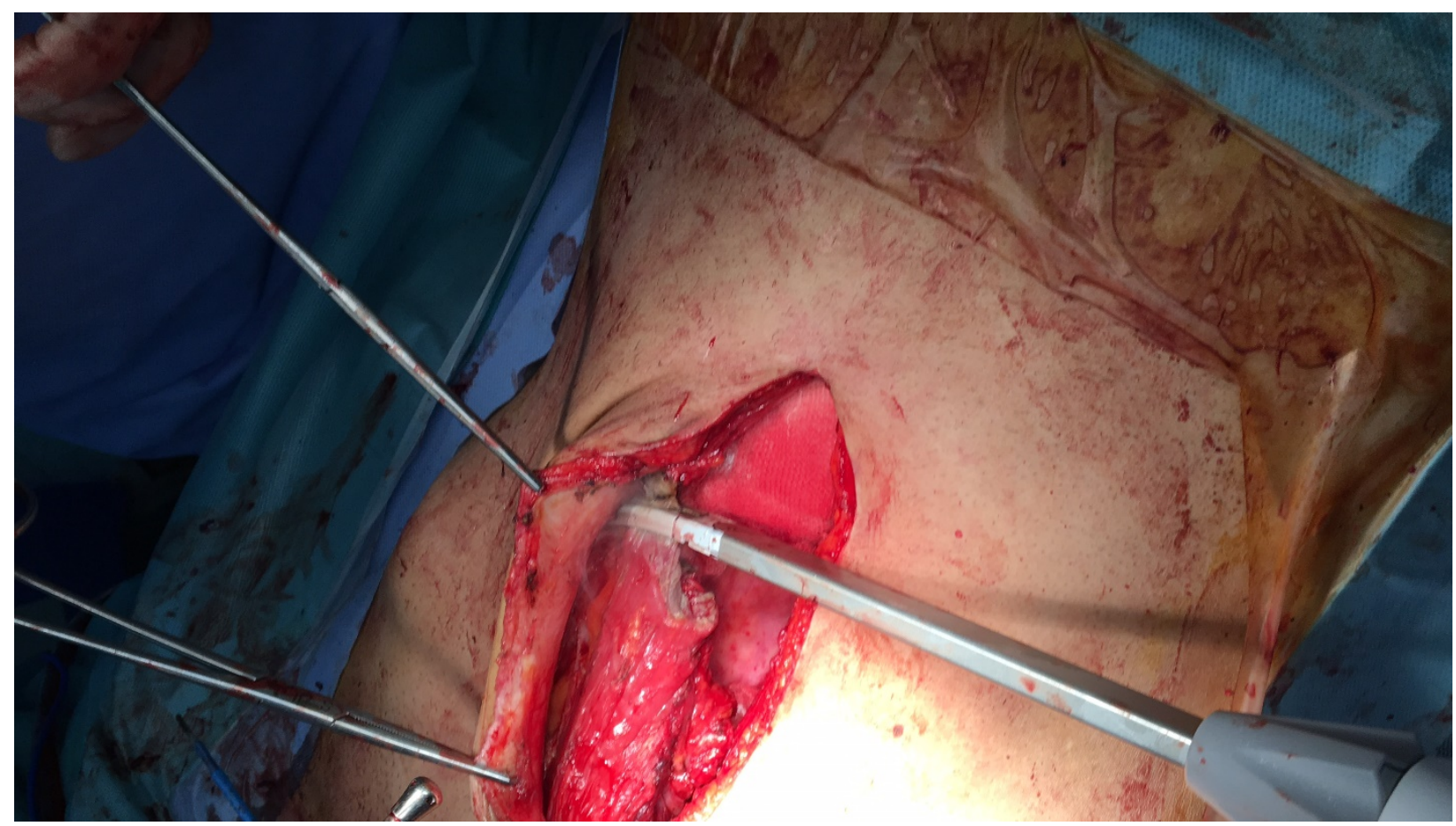

Fig. 2: The inferior part of rectus abdominis muscle was cut just blow the arcuate line and medial to the semi lunar line

midline incision was closed using anterior rectus fascia of both sides and posterior fascia of the left side. Skin closed using 3-0 separate nylon stiches.

\section{Statistical analysis}

For statistical analysis, IBM SPSS statistics 22 (IBM, Inc, New York, USA) was used. For numerical variables means \pm standard deviations and for categorical variables frequencies were reported.

\section{Ethical Approval}

The study protocol was approved by the Ethics Committee of Iran University of Medical Sciences
(Ethical Code: IR.IUMS.REC.1396.921124). An informed consent was obtained from all participants and they were assured regarding data confidentiality. Patients were free to leave the study at any point without affecting their routine care. Helsinki declaration was obeyed in all stages.

\section{RESULTS}

Among 18 participants, $27.8 \%$ were female. The mean age of participants was $58.28 \pm 17.86 \mathrm{yr}$ (minimum of 19 and the maximum of $89 \mathrm{yr}$ ). All patients had distal rectal cancer with sphincter 


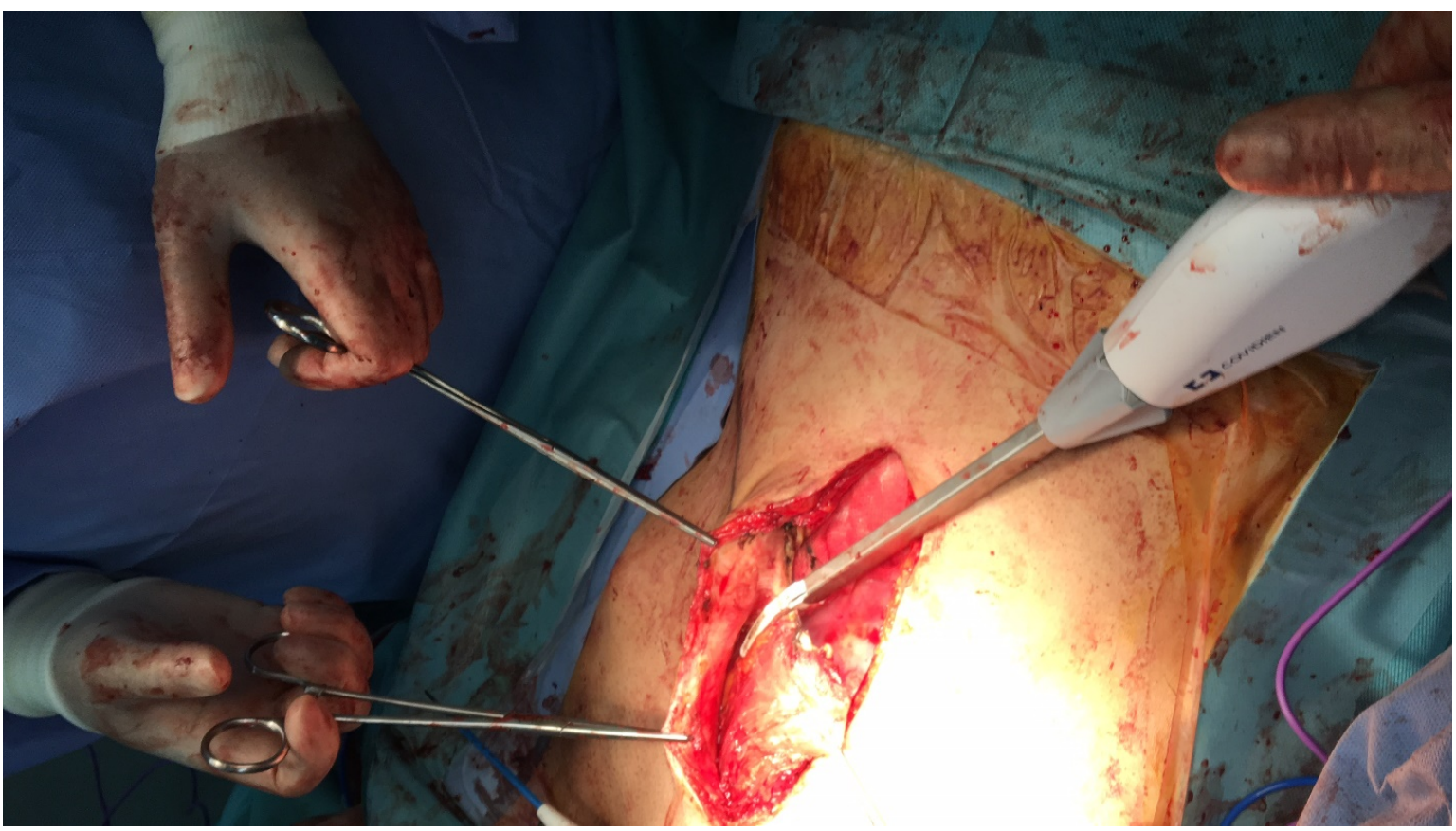

Fig. 3: The inferior part of rectus abdominis muscle flap dissection was continued to the origin which is on the pubis

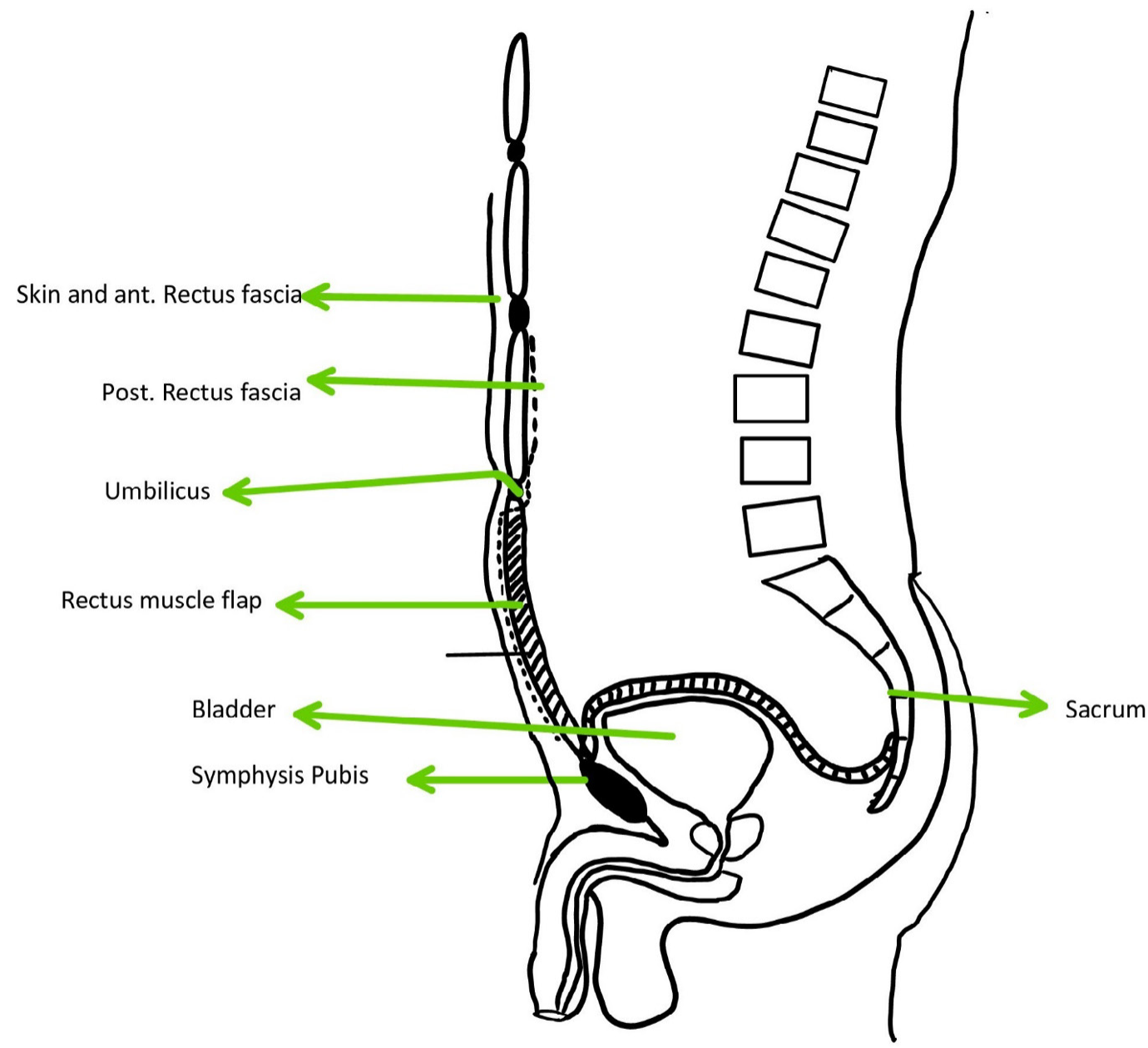

Fig. 4: The inferior part of rectus abdominis flap was fashioned into the perineum 


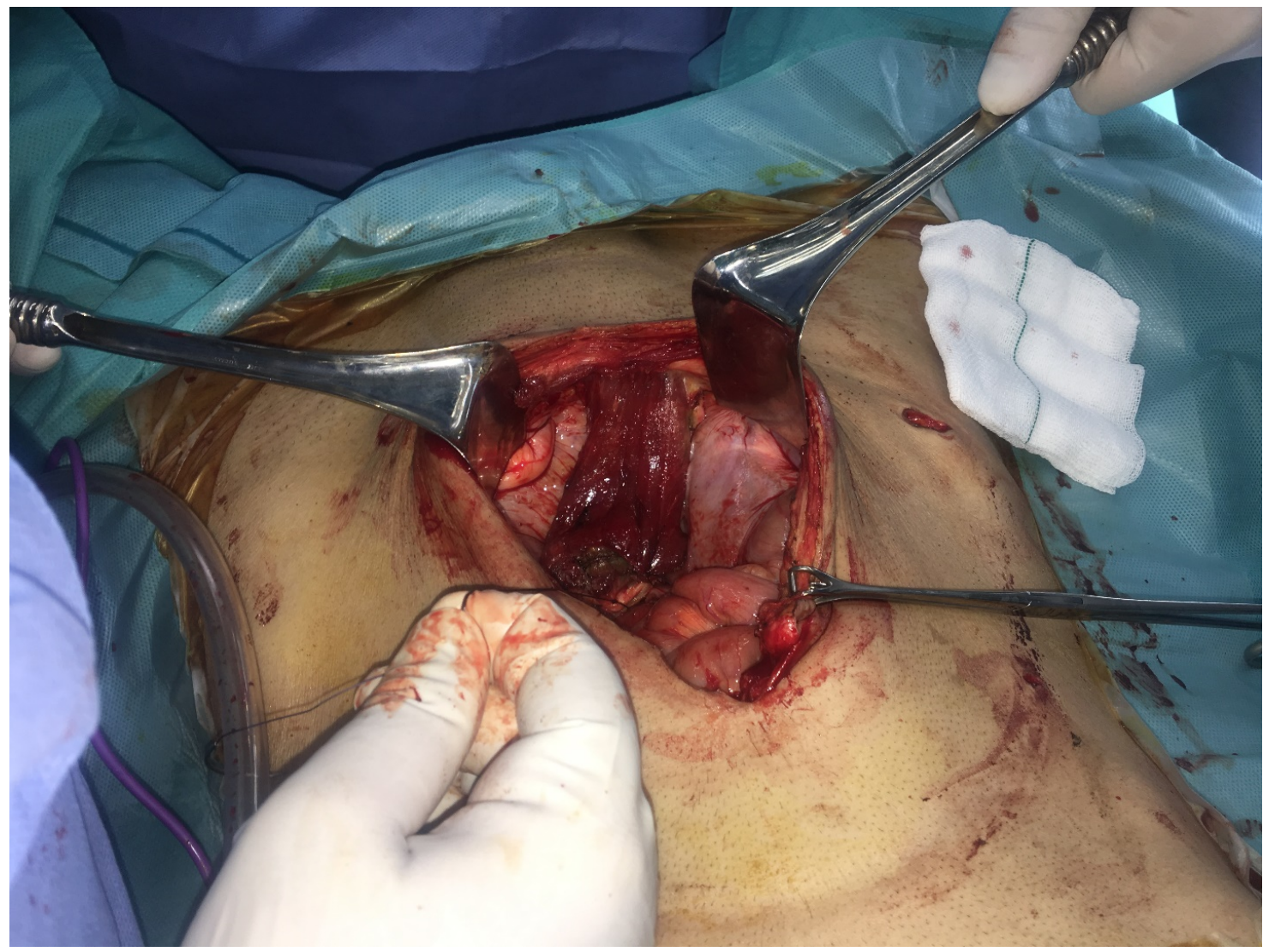

Fig. 5: Multiple layered sutures were used to place the flap without tension

Table 1: The frequency of complications after abdominopelvic resection surgery with inferior part of rectus abdominis muscle flap and before discharge

\begin{tabular}{lcc}
\hline Complications & Frequency & Percent \\
\hline No complications & 13 & 77.8 \\
Wound infection & 1 & 5.6 \\
Dehiscence & 1 & 5.6 \\
Obstruction & 1 & 5.6 \\
Intra-abdominal abscess & 1 & 5.6 \\
\hline
\end{tabular}

involvement (T4a). The pathology of the tumor in all but one of the cases was adenocarcinoma (94.4\%). No patient had distant metastasis. All of the patients received neo-adjuvant chemoradiotherapy.

The mean duration for surgery was $3.30 \pm 0.54$ $\mathrm{h}$ (minimum of 2 and the maximum of $4 \mathrm{~h}$ ). The mean hospital stay after surgery was $6.55 \pm 3.07 \mathrm{~d}$ (minimum of 3 and the maximum of 17 days). It took $1.11 \pm 0.22 \mathrm{~d}$ for patients to get out of bed. The mean duration to start oral nutrition was $2.05 \pm 0.8$ days. A patient was hospitalized for $17 \mathrm{~d}$ to undergo irrigation from the APR defect every other day due to perforated distal rectal cancer.

Post-op complications are listed in Table 1. No flap loss, cellulitis, and prolonged healing was observed.
Of these 18 patients, $2(11.1 \%)$ needed a reoperation after complications (dehiscency, and obstruction). One patient with abscess formation underwent a radiologic drainage and the one with abdominal wound infection was treated conservatively with antibiotics and wound care. In the post-operative period, no mortality was observed. No patient developed deep vein thrombosis or pulmonary emboli, parastomal hernias or urinary tract infection. The frequency of abdominal hernia was nil. The patients were followed for 12 months at 1,3 , 6 and 12 months after discharge. Almost all patients complained about the colostomy and how it affected their quality of life but no questionnaire was filled. In one-month period after surgery no complications 
were seen considering the surgery. At 3 months post-op visit, no complications reported. However, at six visit and 12 months visit, 2 patients (11.2\%) developed a nonfunctioning colostomy and went for reoperation.

\section{DISCUSSION}

Overall complication rate after surgery was $22.4 \%$, among these patients $80 \%$ had received radiotherapy before surgery. No complications were observed for 6 months after surgery, but within 6 to 12 months, 2 patients developed non-functioning colostomy and were re-operated. No mortality was recorded in 12 months follow up after surgery.

Flap reconstruction seems to be a preferred method of reconstruction in variety of surgeries ${ }^{22,23}$ and primary closure might be associated with some complications such as infection or dehiscence ${ }^{24}$.

Sheckter et al. compared three different methods for pelvic floor reconstruction. The rate of complications is higher in V-to-Y advancement group (73.03\%). However, VRAM flap was superior to the other two techniques (rate of complications; 22.2\%). Due to limited sample size in the stated study, a rationale conclusion might not be possible ${ }^{25}$.

Other investigations also verified The VRAM flap safety for abdominopelvic resection with complications up to about $10 \%$. Considering the stage of malignancy, the complication rate could be varied. The overall complication rate of VRAM flaps were $12.96 \%$ to $22.86 \%$ in different stages of rectal cancers $^{26}$. The complication rate in this study which required intervention was $15.2 \%$ after surgery. No patient had flap related complications.

Moreover, no mortality was recorded in the hospital after surgery. This is in line with other studies that evaluated the outcomes of VRAM flaps after APR, and no mortality was reported ${ }^{27}$. Considering that no mortality was recorded, the inferior part of rectus abdominis muscle flap technique has a low mortality rate and comparable to other techniques of reconstruction ${ }^{28}$.

Furthermore, dissection is less in the inferior part of rectus abdominis compared to VRAM flaps, therefore, post-op morbidities, especially abdominal wall ones are less common. Despite the fact, ostomy placement in VRAM is a matter of challenge but the left side is intact in rectus flap ${ }^{9,25}$. However, the muscular bulk in the rectus flap is less compared to VRAM, but it filled the pelvis appropriately. Further studies are recommended to investigate and compare the inferior rectus portion with the VRAM flap method in larger prospective studies.

We had some limitations; 1 ) the sample size of this study was low, so the results of this study cannot be generalized, 2) since it was a cross sectional study the preliminary results cannot be generalized so it is suggested to perform further studies with prospective design 3) the follow up period of this study should be extended.

\section{CONCLUSION}

The inferior part of rectus abdominis muscle flap seems to be a reliable and comparable means of reconstruction after APR surgery with low rate of complications and mortality.

\section{ACKNOWLEDGEMENTS}

This study was funded by the Vice Chancellor for Research \& Technology of Iran University of Medical Sciences under the grant number of 8827 and the ethical code of IR.IUMS.REC.1396.921124.

\section{CONFLICT OF INTEREST}

The authors disclose no conflict of interest.

\section{REFERENCES}

1 Shibata D, Hyland W, Busse P, et al. Immediate reconstruction of the perineal wound with gracilis muscle flaps following abdominoperineal resection and intraoperative radiation therapy for recurrent carcinoma of the rectum. Ann Surg Oncol 1999;6(1):33-7.

2 Chessin DB, Hartley J, Cohen AM, et al. Rectus flap reconstruction decreases perineal wound complications after pelvic chemoradiation and surgery: a cohort study. Ann Surg Oncol 2005 ;12(2):104-10.

3 Chan S, Miller M, Ng R, et al. Use of myocutaneous flaps for perineal closure following abdominoperineal excision of the rectum for adenocarcinoma. Colorectal Dis 2010;12(6):555-60.

4 Howell AM, Jarral OA, Faiz O, Ziprin P, Darzi A, Zacharakis E. How should perineal wounds be closed following abdominoperineal resection in patients post radiotherapy--primary closure or flap repair? Best evidence topic (BET). Int J Surg 2013;11(7):514-7. 
5 Wang ED, Conkling $\mathrm{N}, \mathrm{Xu} \mathrm{X}$, et al. Perineal flap reconstruction following oncologic anorectal extirpation: an outcomes assessment. Plast Reconstr Surg 2015;135(1):176e-84e.

6 Khoo AK, Skibber JM, Nabawi AS, et al. Indications for immediate tissue transfer for soft tissue reconstruction in visceral pelvic surgery. Surgery 2001;130(3):463-9.

7 Baird WL, Hester TR, Nahai F, Bostwick J, 3rd. Management of perineal wounds following abdominoperineal resection with inferior gluteal flaps. Arch Surg 1990;125(11):1486-9.

8 Anthony JP, Mathes SJ. The recalcitrant perineal wound after rectal extirpation. Applications of muscle flap closure. Arch Surg 1990;125(10):1371-6; discussion 6-7.

9 Kolehmainen M, Suominen S, Tukiainen E. Pelvic, perineal and genital reconstructions. Scand J Surg 2013;102(1):25-31.

10 Galandiuk S, Jorden J, Mahid S, McCafferty $\mathrm{MH}$, Tobin G. The use of tissue flaps as an adjunct to pelvic surgery. Am J Surg 2005;190(2):186-90.

11 Persichetti P, Cogliandro A, Marangi GF, et al. Pelvic and perineal reconstruction following abdominoperineal resection: the role of gracilis flap. Ann Plast Surg 2007;59(2):168-72.

12 Burke TW, Morris M, Roh MS, Levenback C, Gershenson DM. Perineal reconstruction using single gracilis myocutaneous flaps. Gynecol Oncol 1995;57(2):221-5.

13 Carey JN, Sheckter CC, Watt AJ, Lee GK. Intraabdominal pedicled rectus abdominis muscle flap for treatment of high-output enterocutaneous fistulae: case reports and review of literature. J Plast Reconstr Aesthet Surg 2013;66(8):1145-8.

14 Touny A, Othman H, Maamoon S, Ramzy S, Elmarakby H. Perineal reconstruction using pedicled vertical rectus abdominis myocutaneous flap (VRAM). J Surg Oncol 2014;110(6):752-7.

15 Kroll SS, Pollock R, Jessup JM, Ota D. Transpelvic rectus abdominis flap reconstruction of defects following abdominal-perineal resection. Am Surg 1989;55(10):632-7.

16 McAllister E, Wells K, Chaet M, Norman J, Cruse W. Perineal reconstruction after surgical extirpation of pelvic malignancies using the transpelvic transverse rectus abdominal myocutaneous flap. Ann Surg Oncol 1994;1(2):164-8.

17 Nelson RA, Butler CE. Surgical outcomes of VRAM versus thigh flaps for immediate reconstruction of pelvic and perineal cancer resection defects. Plast
Reconstr Surg 2009;123(1):175-83.

18 Pantelides NM, Davies RJ, Fearnhead NS, Malata CM. The gluteal fold flap: a versatile option for perineal reconstruction following anorectal cancer resection. J Plast Reconstr Aesthet Surg 2013;66(6):812-20.

19 Windhofer C, Michlits W, Heuberger A, Papp C. Perineal reconstruction after rectal and anal disease using the local fascio-cutaneous-infragluteal flap: A new and reliable technique. Surgery 2011;149(2):28490.

20 Ducic I, Dayan JH, Attinger CE, Curry P. Complex perineal and groin wound reconstruction using the extended dissection technique of the gracilis flap. Plast Reconstr Surg 2008;122(2):472-8.

21 Alemrajabi M, Moradi S, Jahanian S, Banivaheb B, Hemmati N. Inferior part of rectus abdominis muscle flap: A case report. Medical Journal of the Islamic Republic Of Iran 2019;33(1):666-9.

22 Azizi R, Alemrajabi M. Trends in surgical treatment of pilonidal sinus diseases: primary closure or flap? World J Surg 2012;36(7):1713.

23 Azizi R, Alemrajabi M, Naderan M, Shoar S. Efficacy of modified Limberg flap in surgical treatment of infected pilonidal abscess: a case-control study. European Surgery 2014;46(4):144-7.

24 Vahedian J, Jahanian S, Banivaheb B, et al. A New Method for Surgical Abdominal Mass Closure After Abdominal Fascial Dehiscence Using Nasogastric Tube and Hemovac Perforator: A Case-Series Study. World J Surg 2018;42(10):3106-11.

25 Sheckter CC, Shakir A, Vo H, Tsai J, Nazerali R, Lee GK. Reconstruction following abdominoperineal resection (APR): Indications and complications from a single institution experience. J Plast Reconstr Aesthet Surg 2016;69(11):1506-12.

26 Horch RE, Hohenberger W, Eweida A, et al. A hundred patients with vertical rectus abdominis myocutaneous (VRAM) flap for pelvic reconstruction after total pelvic exenteration. Int $J$ Colorectal Dis 2014;29(7):813-23.

27 Hinojosa MW, Parikh DA, Menon R, Wirth GA, Stamos MJ, Mills S. Recent experience with abdominal perineal resection with vertical rectus abdominis myocutaneous flap reconstruction after preoperative pelvic radiation. Am Surg 2009;75(10):995-9.

28 Sunesen KG, Buntzen S, Tei T, Lindegaard JC, Norgaard M, Laurberg S. Perineal healing and survival after anal cancer salvage surgery: 10-year experience with primary perineal reconstruction using the vertical rectus abdominis myocutaneous (VRAM) flap. Ann Surg Oncol 2009;16(1):68-77. 